\title{
NEAR-RINGS OF QUOTIENTS OF ENDOMORPHISM NEAR-RINGS
}

\author{
by MICHAEL HOLCOMBE \\ (Received 8th February 1974)
}

\section{Introduction}

Let $\mathscr{C}$ be a category with finite products and a final object and let $X$ be any group object in $\mathscr{C}$. The set of $\mathscr{C}$-morphisms, $\operatorname{Mor}_{\mathscr{C}}(X, X)$ is, in a natural way, a near-ring which we call the endomorphism near-ring of $X$ in $\mathscr{C}$. Such nearrings have previously been studied in the case where $\mathscr{C}$ is the category of pointed sets and mappings, (6). Generally speaking, if $\Gamma$ is an additive group and $S$ is a semigroup of endomorphisms of $\Gamma$ then a near-ring can be generated naturally by taking all zero preserving mappings of $\Gamma$ into itself which commute with $S$ (see 1). This type of near-ring is again an endomorphism near-ring, only the category $\mathscr{C}$ is the category of $S$-acts and $S$-morphisms (see (4) for definition of $S$-act, etc.).

The question answered in this paper is the following. Under what conditions do endomorphism near-rings of this type have near-rings of quotients which are 2-primitive with d.c.c. on right ideals and an identity? The conditions obtained are described in terms of conditions on the semigroup $S$ and the group $\Gamma$, and are formalised by introducing the concept of a 2-system. 2-primitive near-rings with d.c.c. on right ideals and an identity have been classified in terms of endomorphism near-rings by Wielandt (6) (see also Ramakotaiah (5) and Holcombe (3)).

\section{Terminology}

A near-ring is a set $N$ with two binary operations, addition ( + ) and multiplication (.), such that

(i) $N$ is a group with respect to addition.

(ii) $N$ is a semigroup with respect to multiplication.

(iii) For any $n, n_{1}, n_{2} \in N, n .\left(n_{1}+n_{2}\right)=n . n_{1}+n . n_{2}$.

(iv) If 0 is the additive identity of $N$, then $0 . n=n .0=0$ for all $n \in N$.

A subnear-ring $S$ of a near-ring $N$ is a subset $S$ of $N$, which is a near-ring under the induced binary operations.

A mapping $f: N \rightarrow N_{1}$ of two near-rings $N, N_{1}$ is a near-ring homomorphism if $\left(n+n^{\prime}\right) f=n f+n^{\prime} f ;\left(n . n^{\prime}\right) f=(n f) .\left(n^{\prime} f\right)$ for any $n, n^{\prime} \in N$. 
If $N$ is a near-ring, then an additive group $M$ is an $N$-module, if there exists a mapping $(m, n) \rightarrow m . n$ of $M \times N$ into $M$, such that

(i) $m \cdot\left(n+n_{1}\right)=m \cdot n+m \cdot n_{1}$ for all $m \in M ; n, n_{1} \in N$.

(ii) $m \cdot\left(n \cdot n_{1}\right)=(m \cdot n) \cdot n_{1}$ for all $m \in M ; n, n_{1} \in N$.

A mapping $\psi: M \rightarrow M_{1}$ (where $M$ and $M_{1}$ are $N$-modules) is called an $N$-homomorphism if

$$
\begin{array}{ll}
\left(m+m^{\prime}\right) \psi=m \psi+m^{\prime} \psi & \text { for any } m, m^{\prime} \in M \\
(m n) \psi=(m \psi) n & \text { for any } m \in M ; n \in N .
\end{array}
$$

An $N$-submodule $M^{\prime}$ of an $N$-module $M$ is the kernel of an $N$-homomorphism from $M$, so that $M^{\prime} \triangleleft M$ and $\left(m+m^{\prime}\right) . n-m . n \in M^{\prime}$ for all $m \in M, m^{\prime} \in M^{\prime}, n \in N$.

An $N$-subgroup $M^{\prime \prime}$ of an $N$-module $M$ is an additive subgroup $M^{\prime \prime}$ of $M$ such that $m^{\prime \prime} . n \in M^{\prime \prime}$ for all $m^{\prime \prime} \in M^{\prime \prime}, n \in N$.

A near-ring $N$ is clearly an $N$-module.

A right ideal of $N$ is an $N$-submodule of the $N$-module $N$.

A right $N$-subgroup of $N^{+}$is an $N$-subgroup of the $N$-module $N$.

An $N$-module $M$ is of type 2 if $M$ possesses no proper, non-trivial $N$-subgroups and $M N=\{m n \mid m \in M ; n \in N\} \neq\{0\}$.

Let $S$ be a subset of an $N$-module $M$, we define

$$
(S)_{r}=\{n \in N \mid s n=0 \text { for all } s \in S\}
$$

and call this the right annihilator of $S$ (in $N)$. Clearly this is a right ideal of $N$.

A near-ring $N$ is 2-primitive if there exists an $N$-module $M$ of type 2 such that $(M)_{r}=(0)$.

For any near-ring $N$, define $J_{2}(N)$ to be the intersection of the right annihilators of all $N$-modules of type 2 , with the convention that $J_{2}(N)=N$ if $N$ possesses no $N$-modules of type 2 .

A near-ring $N$ is 2-prime if $a N b=(0) \Rightarrow a=0$ or $b=0(a, b \in N)$.

A near-ring satisfies the descending chain condition (d.c.c.) on right ideals if, given a chain of right ideals in the near-ring,

$$
R_{1} \supsetneq R_{2} \supsetneq \cdots \supsetneq R_{p} \supsetneq \cdots
$$

then there exists an integer $q$ with $R_{q}=R_{q+1}=\ldots$

A near-ring satisfies the ascending chain condition (a.c.c.) on right annihilators if given a chain of right ideals of the near-ring, which are right annihilators,

$$
\left(Z_{1}\right)_{r} \subsetneq\left(Z_{2}\right)_{r} \varsubsetneqq \cdots \varsubsetneqq\left(Z_{p}\right)_{r} \varsubsetneqq \cdots
$$

then there exists an integer $q$ with $\left(Z_{q}\right)_{r}=\left(Z_{q+1}\right)_{r} \ldots$

An element $n$ of a near-ring $N$ is a regular element if

and

$$
n_{1} n=n_{2} n \Rightarrow n_{1}=n_{2} \quad\left(n_{1}, n_{2} \in N\right)
$$

$$
n n_{3}=n n_{4} \Rightarrow n_{3}=n_{4} \quad\left(n_{3}, n_{4} \in N\right) \text {. }
$$




\section{QUOTIENTS OF ENDOMORPHISM NEAR-RINGS}

A near-ring $N$ has a near-ring of right quotients $Q$ if

(i) $N$ can be embedded (as a near-ring) in $Q$, and $Q$ has an identity;

(ii) $x$ is a regular element of $N$ then $\exists y \in Q$ such that $x \cdot y=y \cdot x=1_{Q}$ (we write $y=x^{-1}$ );

(iii) $q \in Q$ then $q=n x^{-1}$ where $n \in N$ and $x$ is a regular element of $N$.

Let $\Gamma$ be an additive group and $S$ a semigroup of endomorphisms of $\Gamma$. We define $\operatorname{Map}_{S}(\Gamma)$ to be the set of mappings $\left\{n \mid n: \Gamma \rightarrow \Gamma ;\left(0_{\Gamma}\right) \cdot n=0_{\Gamma}\right.$; $\gamma s n=\gamma n s, \forall \gamma \in \Gamma, \forall s \in S\}$. Then $\operatorname{Map}_{s}(\Gamma)$ is a near-ring (with a multiplicative identity), called the endomorphism near-ring of $\Gamma$ in the category of $S$-acts and S-maps.

\section{2-Systems}

Theorem 2.1. Suppose that $\Gamma$ is an additive group, and $S$ is a multiplicative semigroup of endomorphisms of $(\Gamma,+)$, which includes the identity endomorphism, but not the zero endomorphism. Suppose that $S$ is left and right cancellative, left and right reversible and for all $s \in S, \gamma s=0 \Rightarrow \gamma=0,(\gamma \in \Gamma)$. Then $S$ has a group $G$ of left quotients, and $G$ acts as a group of automorphisms on an additive group $\Delta$.

Proof. The existence of $G$ is a standard result (2).

Consider $\Gamma \times S$. Let $(\gamma, s),\left(\gamma_{1}, s_{1}\right) \in \Gamma \times S$, define the relation $\sim$ by $(\gamma, s) \sim\left(\gamma_{1}, s_{1}\right) \Leftrightarrow \exists a, b, \in S$ such that $s b=s_{1} a$ and $\gamma b=\gamma_{1} a$.

It is a fairly mechanical procedure to verify that $\sim$ is well defined and an equivalence relation (see (6)). Partitioning $\Gamma \times S$ into equivalence classes we write $\gamma / s$ to represent the equivalence class containing $(\gamma, s)$. Let

$$
\Delta=\{\gamma / s \mid \gamma \in \Gamma, s \in S\}
$$

We show that $\Delta$ is an additive group and $G$ is a group of automorphisms of $\Delta$.

Let $\gamma / s, \gamma^{\prime} / s^{\prime} \in \Delta$ and define $\gamma / s+\gamma^{\prime} / s^{\prime}=\left(\gamma a+\gamma^{\prime} b\right) / m$ where $a, b \in S$, such that $s^{\prime} b=s a=m$. This operation is well defined. For suppose $\left(\gamma_{1}, s_{1}\right) \sim(\gamma, s)$ and $\left(\gamma_{1}^{\prime}, s_{1}^{\prime}\right) \sim\left(\gamma^{\prime}, s^{\prime}\right)$; we show that $\gamma_{1} / s_{1}+\gamma_{1}^{\prime} / s_{1}^{\prime}=\gamma / s+\gamma^{\prime} / s^{\prime}$. Let $\alpha, \beta \in S$ such that $s \alpha=s_{1} \beta$ and $\gamma \alpha=\gamma_{1} \beta$. Also let $\lambda, \mu \in S$ such that $s^{\prime} \lambda=s_{1}^{\prime} \mu$ and $\gamma^{\prime} \lambda=\gamma_{1}^{\prime} \mu$. Then $\gamma_{1} / s_{1}+\gamma_{1}^{\prime} / s_{1}^{\prime}=\left(\gamma_{1} x+\gamma_{1}^{\prime} y\right) / s_{1} x$, where $x, y \in S$ such that $s_{1}^{\prime} x=s_{1}^{\prime} y$. Choose $e, f \in S$ with $s_{1} x f=$ sae. Then sae $=s^{\prime} b e, s_{1} x f=s_{1}^{\prime} y f$. Therefore $s^{\prime} b e=s_{1}^{\prime} y f$. Now there exist $k, h \in S$ such that $a e k=\alpha h$. Then saek $=s \alpha h=s_{1} \beta h=s_{1} x f k$; thus $\beta h=x f k . \quad \gamma a e k=\gamma \alpha h=\gamma_{1} x f k$. Then $\left(\right.$ yae $\left.-\gamma_{1} x f\right) k=0 \Rightarrow$ yae $=\gamma_{1} x f$. Also $\gamma^{\prime} b e l=\gamma^{\prime} \lambda m^{\prime}$, where $l, m^{\prime} \in S$ such that bel $=\lambda m^{\prime}$. Now $s^{\prime} b e l=s a e l=s_{1}^{\prime} y f l=s^{\prime} \lambda m^{\prime}$. Then $s_{1}^{\prime} y f l=s_{1}^{\prime} \mu m^{\prime} \Rightarrow y f l=\mu m^{\prime}$ and $\gamma^{\prime} b e l=\gamma^{\prime} \lambda m^{\prime}=\gamma_{1}^{\prime} \mu m^{\prime}=\gamma_{1}^{\prime} y f l \Rightarrow \gamma^{\prime} b e=\gamma_{1}^{\prime} y f$. Since $e$ and $f$ are endomorphisms of $\Gamma,\left(\gamma a+\gamma^{\prime} b\right) e=\gamma a e+\gamma^{\prime} b e=\gamma_{1} x f+\gamma_{1}^{\prime} y f=\left(\gamma_{1} x+\gamma_{1}^{\prime} y\right) f$ : but sae $=s_{1} x f$; hence $\left(\gamma a+\gamma^{\prime} b, s a\right) \sim\left(\gamma_{1} x+\gamma_{1}^{\prime} y, s_{1} x\right)$. Thus addition is well defined, and the group axioms are satisfied. If $\gamma / s \in \Delta$ and $g \in G$, with $g=r / s_{1}\left(r, s_{1} \in S\right)$ 
define $(\gamma / s) \cdot g=\gamma u_{1} /\left(s_{1} b_{1}\right)$, where $g=r / s_{1}$ and $u_{1}, b_{1} \in S$ such that $s u_{1}=r b_{1}$. It may be checked that this is a well-defined operation and $g$ is an automorphism of $(\Delta,+)$, and $G$ acts faithfully on $\Delta$. For example, let $\gamma / s, \gamma^{\prime} / s^{\prime}, \in \Delta$ and $g=r / s_{1} \in G$. Then $\left(\gamma / s+\gamma^{\prime} / s^{\prime}\right)\left(r / s_{1}\right)=\left(\left(\gamma a+\gamma^{\prime} b\right) / s a\right)\left(r / s_{1}\right)$, where $s a=s^{\prime} b$ so

$$
\left(\gamma / s+\gamma^{\prime} / s^{\prime}\right)\left(r / s_{1}\right)=\left(\gamma a+\gamma^{\prime} b\right)\left(u_{1} /\left(s_{1} b_{1}\right)\right)=\left(\gamma a u_{1}+\gamma^{\prime} b u_{1}\right) /\left(s_{1} b_{1}\right),
$$

where $s a u_{1}=r b_{1}$. Now $(\gamma / s)\left(r / s_{1}\right)=\left(\gamma u_{2}\right) /\left(s_{1} b_{2}\right)$, where $s u_{2}=r b_{2}$, and $\left(\gamma^{\prime} / s^{\prime}\right)\left(r / s_{1}\right)=\left(\gamma^{\prime} u_{3}\right) /\left(s_{1} b_{3}\right)$, where $s^{\prime} u_{3}=r b_{3}$. Then

$$
(\gamma / s)\left(r / s_{1}\right)+\left(\gamma^{\prime} / s^{\prime}\right)\left(r / s_{1}\right)=\left(\gamma u_{2} c+\gamma^{\prime} u_{3} d\right) /\left(s_{1} b_{2} c\right),
$$

where $s_{1} b_{2} c=s_{1} b_{3} d$, i.e. $b_{2} c=b_{3} d$. Choose $x, y \in S$ such that $s_{1} b_{2} c x=s_{1} b_{1} y$. Then $b_{2} c x=b_{1} y=b_{3} d x$ and $s u_{2} c x=r b_{2} c x=r b_{3} d x=r b_{1} y=s a u_{1} y$; hence $u_{2} c x=a u_{1} y \Rightarrow \gamma u_{2} c x=\gamma a u_{1} y$. Also $s^{\prime} u_{3} d x=r b_{3} d x=s^{\prime} b u_{1} y$; so $u_{3} d x=b u_{1} y$ and $\gamma^{\prime} u_{3} d x=\gamma^{\prime} b u_{1} y$. Then $\left(\gamma a u_{1}+\gamma^{\prime} b u_{1}\right) y=\gamma a u_{1} y+\gamma^{\prime} b u_{1} y=\left(\gamma u_{2} c+\gamma^{\prime} u_{3} d\right) x$, i.e. $\left(\gamma a u_{1}+\gamma^{\prime} b u_{1}, s_{1} b_{1}\right) \sim\left(\gamma u_{2} c+\gamma^{\prime} u_{3} d, s_{1} b_{2} c\right)$. In this way we see that $G$ is a group of endomorphisms of $\Delta$, and is in fact easily seen to be a group of automorphisms of $\Delta$.

Lemma 2.2. In the terminology of 2.1, $G$ is a group of regular automorphisms of $\Delta$, if and only if, for every $0 \neq \gamma \in \Gamma, \gamma s_{1}=\gamma s_{2} \Rightarrow s_{1}=s_{2},\left(s_{1}, s_{2} \in S\right)$.

Proof. (i) Suppose that $G$ is a group of regular automorphisms. Let $\gamma \in \Gamma ; s_{1} \in S, s_{2} \in S$ with $\gamma s_{1}=\gamma s_{2}$ and $s_{1} \neq s_{2}$. Then $(\gamma / 1)\left(s_{1} / s_{2}\right)=(\gamma \cdot a) /\left(s_{2} b\right)$, where $1 . a=s_{1} b$. Hence $(\gamma / 1)\left(s_{1} / s_{2}\right)=\left(\gamma s_{1} b\right) /\left(s_{2} b\right)=\left(\gamma s_{2} b\right) /\left(s_{2} b\right)=\gamma$. Since $g=s_{1} / s_{2} \in G$, we have found an element $\gamma \in \Gamma$ and $g \in G$ such that $\gamma g=\gamma$. But $g$ is regular; so $\gamma=0$.

(ii) Suppose that $\gamma / s \in \Delta, r / s_{1} \in G$ and $\gamma \neq 0$, and $(\gamma / s)\left(r / s_{1}\right)=\gamma / s$. Then $\gamma / s=\gamma u_{1} /\left(s_{1} b_{1}\right)$, where $r b_{1}=s u_{1}$. There exist $u_{2}, b_{2} \in S$ such that $\gamma u_{2}=\gamma u_{1} b_{2}$ and $s u_{2}=s_{1} b_{1} b_{2}$. Now $\gamma \neq 0 \Rightarrow u_{2}=u_{1} b_{2}$ and thus

$$
s_{1} b_{1} b_{2}=s u_{2}=s u_{1} b_{2} \Rightarrow s_{1} b_{1}=s u_{1}=r b_{1} \Rightarrow s_{1}=r .
$$

Thus $r / s_{1}$ is the identity automorphism of $\Delta$, and $G$ is a group of regular automorphisms of $\Delta$.

Lemma 2.3. In the terminology of Theorem 2.1 if $\Gamma=\{0\} \cup\left\{\bigcup_{i=1}^{p} \gamma_{i} S\right\}$ for suitable $\gamma_{1}, \gamma_{2}, \ldots, \gamma_{p} \in \Gamma$, such that $\gamma_{1} S \cap \gamma_{j} S=\varnothing$ for $i \neq j$, then $\Delta$ has $p$ orbits under the action of $G$.

Proof. Let $\delta \in \Delta, \delta \neq 0$. Then $\delta=\gamma / s$ for some $\gamma \in \Gamma, s \in S$, and $\gamma=\gamma_{i} s_{i}$ for some $s_{i} \in S$, and some $i \in\{1,2, \ldots, p\}$. Then $\delta=\left(\gamma_{i} s_{i}\right) / s=\gamma_{i}\left(s_{i} / s\right) \in \bigcup_{i=1}^{p} \gamma_{i} G$. Thus $\Delta=\left\{\bigcup_{i=1}^{p} \gamma_{i} G\right\} \cup\{0\}$. Now suppose that $\delta^{\prime} \neq 0$ and $\delta^{\prime} \in \gamma_{i} G \cap \gamma_{j} G$ for $i \neq j$. Let $\delta^{\prime}=\gamma_{i}(r / s)=\gamma_{j}(y / z)$, where $r, s, y, z \in S$. There exist $\alpha, \beta \in S$ such that $s \alpha=z \beta=m$ (say); so we have $\delta^{\prime}=\left(\gamma_{i} r\right) / s=\left(\gamma_{j} y\right) / z$ and 
$\delta^{\prime} m=\left(\gamma_{i} r / s\right)(m / 1)=\gamma_{i} r u_{1} / b_{1}$, where $u_{1}, b_{1} \in S$ and $m b_{1}=s u_{1}$, i.e. $s \alpha b_{1}=s u_{1}$, i.e. $\alpha b_{1}=u_{1}$. Thus $\delta^{\prime} m=\left(\gamma_{i} r \alpha b_{1}\right) / b_{1}=\gamma_{i} r \alpha$. Also $\delta^{\prime} m=\gamma_{j} y u_{2} / b_{2}$, where $u_{2}, b_{2} \in S$ and $m b_{2}=z u_{2}$, i.e. $\beta b_{2}=u_{2}$. Thus $\delta^{\prime} m=\gamma_{j} y \beta=\gamma_{i} r \alpha$; which implies that $\gamma_{j} S \cap \gamma_{i} S \neq \varnothing$, a contradiction.

Definition. A system $(\Gamma, S)$ satisfying the hypothesis of Theorem 2.1 will be called a 2-system, if and only if for every $0 \neq \gamma \in \Gamma, \gamma s_{1}=\gamma s_{2},\left(s_{1}, s_{2} \in S\right)$.

Theorem 2.4. If $(\Gamma, S)$ and $\left(\Gamma_{1}, S_{1}\right)$ are 2-systems, then $\left(\Gamma \oplus \Gamma_{1}, S \times S_{1}\right)$ is a Q-system.

Proof. $S \times S_{1}$ is a semigroup of monomorphisms of $\Gamma \oplus \Gamma_{1}$ in the natural way. Clearly $S \times S_{1}$ is left and right cancellative, left and right reversible. Let $\left(\gamma, \gamma_{1}\right) \in \Gamma \oplus \Gamma_{1}$ with $\left(\gamma, \gamma_{1}\right) \neq\left(0,0_{1}\right)$ and $\left(\gamma, \gamma_{1}\right)\left(s, s_{1}\right)=\left(\gamma, \gamma_{1}\right)\left(s^{\prime}, s_{1}^{\prime}\right)$ for $s, s^{\prime} \in S ; s_{1}, s_{1}^{\prime} \in S_{1}$. Then $\gamma s=\gamma s^{\prime} \Rightarrow s=s^{\prime}$ and $\gamma_{1} s_{1}=\gamma_{1} s_{1}^{\prime} \Rightarrow s_{1}=s_{1}^{\prime}$. Finally, if $\Gamma=\gamma_{1} S \cup \gamma_{2} S \cup \ldots \cup \gamma_{p} S \cup\{0\}$ and $\Gamma_{1}=\gamma_{11} S_{1} \cup \gamma_{12} S_{1} \cup \ldots \cup \gamma_{1 q} S_{1} \cup\left\{0_{1}\right\}$ then $\Gamma \oplus \Gamma_{1}=\bigcup_{t=1}^{m} \delta_{t}\left(S \times S_{1}\right) \cup\left\{\left(0,0_{1}\right)\right\}$, where $m=p q+p+q$ and the $\delta_{t}$ are elements of the form $\left(\gamma_{i}, 0_{1}\right),\left(0, \gamma_{1 j}\right),\left(\gamma_{i}, \gamma_{1 j}\right)$. Let

$$
\left(\gamma, \gamma^{\prime}\right) \in\left(\lambda_{i}, \lambda_{1 j}\right)\left(S \times S_{1}\right) \cap\left(\lambda_{l}, \lambda_{1 m}\right)\left(S \times S_{1}\right)
$$

for some $\lambda_{i}, \lambda_{l} \in\left\{0, \gamma_{1}, \gamma_{2}, \ldots, \gamma_{p}\right\}$ and $\lambda_{1 j}, \lambda_{1 m} \in\left\{0_{1}, \gamma_{11}, \gamma_{12}, \ldots, \gamma_{1 q}\right\}$. Suppose $\left(\gamma, \gamma^{\prime}\right)=\left(\lambda_{i} s, \lambda_{1 j} s_{1}\right)=\left(\lambda_{l} s^{\prime}, \lambda_{1 m^{m}} s_{1}\right)$ then $\lambda_{i} s=\lambda_{l} s^{\prime}$ and $\lambda_{1 j} s_{1}=\lambda_{1 m} s_{1}^{\prime}$. Clearly $\lambda_{i}=\lambda_{1}$ and $\lambda_{1 j}=\lambda_{1 \mathrm{~m}}$. This proves the result.

Examples. Let $\Gamma$ be the additive group of integers and $S$ the semigroup of positive integers under multiplication. Then $(\Gamma, S)$ is a 2 -system if we define $(\gamma) s=\gamma s$ for all $\gamma \in \Gamma, s \in S$. Also let $\Gamma$ be any finite group and $S=$ identity automorphism $\}$ then $(\Gamma, S)$ is a 2 -system.

\section{The near-rings associated with 2 -systems}

To each 2 -system $(\Gamma, S)$, we have associated an additive group $\Delta$ and a group $G$ of regular automorphisms of $\Delta$ admitting only finitely many orbits on $\Delta$. We now define two near-rings in a natural way. Let $N=\operatorname{Map}_{S}(\Gamma)$ and $Q=\operatorname{Map}_{G}(\Delta)$. We can imbed $N$ in $Q$ in a natural way. Let $n \in N$; define $\bar{n}: \Delta \rightarrow \Delta$ by $0 . \bar{n}=0$ and $\left[\gamma_{i}(r / s)\right] \bar{n}=\left(\gamma_{i} n\right)(r / s)$, where $\gamma_{i}(r / s)$ is a typical non-zero element of $\Delta$, and $\Delta=\{0\} \cup\left(\bigcup_{i=1}^{p} \gamma_{i} G\right)$. It is not difficult to see that $\bar{n} \in Q$. Define a map $\xi: N \rightarrow Q$ by $n \xi=\bar{n}, \forall n \in N$. Let $n, n_{1} \in N$; then $\left(n+n_{1}\right) \xi={\overline{n+n_{1}}}_{1}$. For any $\delta \in \Delta, \delta \neq 0$,

$$
\begin{aligned}
\delta\left(\overline{n+n_{1}}\right) & =\gamma_{i}(a / b)\left(\overline{n+n_{1}}\right)=\gamma_{i}\left(n+n_{1}\right) \cdot(a / b)=\left(\gamma_{i} n+\gamma_{i} n_{1}\right) \cdot(a / b) \\
& =\gamma_{i}\left(n \cdot a / b+n_{1} \cdot a / b\right)=\delta n+\delta n_{1} .
\end{aligned}
$$


Also $\delta\left(\overline{n . n}_{1}\right)=\left(\gamma_{i}(a / b)\right)\left(\overline{n n}_{1}\right)=\left(\gamma_{i} n n_{1}\right) \cdot(a / b)$. Let $\gamma_{i} n n_{1}=\gamma_{j} r_{1}$, for some $j \in\{1, \ldots, p\}$ and $r_{1} \in S$, so that $\delta\left(\overline{n n_{1}}\right)=\gamma_{j} r_{1}(a / b)$. Now

$$
\delta\left(\bar{n} \cdot \bar{n}_{1}\right)=\gamma_{1} n \cdot(a / b) \bar{n}_{1}=\gamma_{k} r_{2}(a / b) \bar{n}_{1},
$$

where $\gamma_{i} n=\gamma_{k} r_{2}$ for some $k \in\{1, \ldots, p\}$ and $r_{2} \in S$. Then

$$
\delta\left(\bar{n} \cdot \bar{n}_{1}\right)=\gamma_{k} \cdot n_{1}\left(r_{2} \cdot a / b\right)
$$

Let $\gamma_{k} n_{1}=\gamma_{l} r_{3}$ for some $l \in\{1, \ldots, p\}$ and $r_{3} \in S$, then

$$
\gamma_{i} n n_{1}=\gamma_{j} r_{1}=\left(\gamma_{k} r_{2}\right) n_{1}=\gamma_{l} r_{3} r_{2}
$$

Hence $\delta\left(\bar{n} \cdot \bar{n}_{1}\right)=\gamma_{1} r_{3} r_{2} \cdot(a / b)=\gamma_{j} r_{1} \cdot(a / b)=\delta\left(\bar{n} \bar{n}_{1}\right)$. Thus $\xi$ is a near-ring homomorphism. If $n \in \operatorname{ker} \xi$ then $\bar{n}=0$, and $\Delta \bar{n}=0$. Thus if $0 \neq \delta \in \Delta$, and $\delta=\gamma_{i}(a / b)$, then $\left[\gamma_{i}(a / b)\right] \bar{n}=\gamma_{i} n(a / b)=0$. In particular, $\gamma_{i} n=0$ and this is clearly true for $i=1, \ldots, p$. Hence $n$ is the zero mapping and $\xi$ is bijective. We have

Theorem 3.1. If $(\Gamma, S)$ is a 2-system, and $\Delta$ and $G$ the groups constructed by Theorem 2.1, then $\operatorname{Map}_{S}(\Gamma)$ may be imbedded in $\operatorname{Map}_{G}(\Delta)$.

Remark. The near-ring $Q=\operatorname{Map}_{G}(\Delta)$ is a 2-primitive near-ring with identity and descending chain condition on right ideals (see (3)). The remainder of this section will show that $Q$ is a near-ring of right quotients of $N$.

Let $I=\{1,2, \ldots, p\}$. Suppose $n \in N$ and for any $k \in I$ define

$$
I_{k}(n)=\left\{i \in I \mid \gamma_{i} n \in \gamma_{k} S\right\}
$$

It is clear that $I_{k}(n)$ may be empty and that if $l \in I$ with $k \neq l$, then

$$
I_{k}(n) \cap I_{l}(n)=\varnothing \text {. }
$$

Lemma 3.2. If $n$ is a regular element of $N$, then each $I_{k}(n)$ contains one element for any $k \in I$.

Proof. If $I_{k}(n)=\varnothing$ for some $k \in I$, define $n_{1}$ so that

$$
\begin{aligned}
& \gamma_{i} s n_{1}=\gamma_{i} s \text { for all } i \in I \text { with } i \neq k \text { and any } s \in S \\
& \gamma_{k} s n_{1}=0 \text { for all } s \in S \text { and } 0 n_{1}=0 .
\end{aligned}
$$

Then $n_{1} \in N, \quad \gamma_{i} n .1=\gamma_{i} n n_{1} \quad(i \neq k)$ and $\gamma_{k} n .1=\gamma_{j} s=\gamma_{j} s n_{1}=\gamma_{k} n n_{1}$, where $\gamma_{k} n=\gamma_{j} s$ for some $j \in I \backslash\{k\}$ and $s \in S$. Thus $n .1=n . n_{1} \Rightarrow n_{1}=1$ which contradicts $\gamma_{k} n_{1}=0$. Thus $I_{k}(n) \neq \varnothing$ for any $k \in I$.

Theorem 3.3. If $n$ is a regular element of $N$, then there exists $q \in Q$ such that $\bar{n} \cdot q=q \cdot \bar{n}=1_{Q}$.

Proof. Let $\gamma_{i} n=\gamma_{j_{i}} . s_{1}$ where $j_{i} \in I ; s_{i} \in S ; i=1, \ldots, p$.

The integers $j_{1}, \ldots, j_{p}$ are a permutation of $1, \ldots, p$ by Lemma 3.2 ; let this permutation be denoted by $\pi$. Thus $j_{i}=\pi(i), i \in I$. Let $s_{i}^{-1}$ be the inverse of $s_{i}$ in $G(i \in I)$. Define $q: \Delta \rightarrow \Delta$ by $0 q=0$ and $\left(\gamma_{\pi(i)} g_{i}\right) q=\gamma_{i} s_{i}^{-1} g_{i}$ for 
$i \in I, g_{i} \in G$. Let $\delta \in \Delta, g \in G$ and suppose that $\delta=\gamma_{\pi(l)} \cdot g_{1}$ for some $l \in I, g_{1} \in G$. Then

$$
(\delta g) q=\left(\gamma_{\pi(l)} \cdot g_{1} g\right) q=\gamma_{i} s_{i}^{-1}\left(g_{1} \cdot g\right)=\left(\gamma_{i} s_{i}^{-1} g_{1}\right) g=\left(\gamma_{\pi(l)} g_{1}\right) q g=\delta q g .
$$

Hence $q \in Q$.

Now let $\delta^{\prime} \in \Delta$ with $\delta^{\prime} \neq 0$, and $\delta^{\prime}=\gamma_{i} g_{i}=\gamma_{i}\left(r_{i} / t_{i}\right)$, where $i \in I, r_{i}, t_{i} \in S$. Then $\delta^{\prime} \bar{n} q=\left(\gamma_{i}\left(r_{i} / t_{i}\right) \bar{n} q=\left(\gamma_{i} n\right)\left(r_{i} / t_{i}\right) q=\left(\gamma_{\pi(i)} s_{i}\right)\left(r_{i} / t_{i}\right) q=\gamma_{i} s_{i}^{-1} s_{i}\left(r_{i} / t_{i}\right)=\delta^{\prime}\right.$.

Hence $\bar{n} q=1_{Q}$. Also $\delta^{\prime} q \bar{n}=\left(\gamma_{i}\left(r_{i} / t_{i}\right)\right) q \bar{n}=\gamma_{\pi(k)}\left(r_{i} / t_{i}\right) q \bar{n}$, where $\pi(k)=i$. Then

$$
\delta^{\prime} q \bar{n}=\gamma_{k} s_{k}^{-1}\left(r_{i} / t_{i}\right) \bar{n}=\gamma_{k} n s_{k}^{-1}\left(r_{i} / t_{i}\right) \text {. }
$$

Now $\gamma_{k} n=\gamma_{\pi(k)} s_{k}$ and so $\delta^{\prime} q \bar{n}=\delta^{\prime}$. Thus $\bar{n} q=q \bar{n}=1_{Q}$. Therefore we can invert the imbedded regular elements of $N$ in $Q$.

Theorem 3.4. If $x$ is an arbitrary non-zero element of $Q$, then there exist $\theta, n_{1} \in N$, with $\theta$ regular in $N$ such that $x=\bar{n}_{1} \theta^{-1}$, where $\theta^{-1}$ is the inverse in $Q$ of the element $\theta$.

We first need the following lemma, which is proved by a standard induction argument.

Lemma 3.5. Let $r_{1}, \ldots, r_{\sigma}, t_{1}, \ldots, t_{\sigma} \in S$, then there exist $m \in S$ and $h_{1}, \ldots, h_{\sigma} \in S$ such that $m r_{i}=h_{i} t_{i}$ for $i=1, \ldots, \sigma$.

Proof of Theorem 3.4. Let $x \in Q$, with $x \neq 0$. Put $X=\left\{\alpha \in I \mid \gamma_{\alpha} x=0\right\}$. Suppose that $x: \gamma_{i} \rightarrow \gamma_{j} g_{i}, i \in I \backslash X, j_{i} \in I, g_{i} \in G$. We have that $x: \gamma_{\alpha} \rightarrow 0$ for $\alpha^{*} \in X$. For any $k \in I$, put $I_{k}^{*}(x)=\left\{i \in I \mid \gamma_{i} x \in \gamma_{k} G\right\}$.

Some of these $I_{k}^{*}(x)$ may be empty. If $v \in I_{k}^{*}(x)$ for some $k \in I$ then $\gamma_{v} x=\gamma_{k} g_{k v}$ say, $\left(g_{k v} \in G\right)$ and so $\gamma_{v} x=\gamma_{k}\left(r_{k v} / t_{k v}\right)$ for suitable $r_{k v}, t_{k v} \in S$. From Lemma 3.5, there exist $m_{k} \in S$ and $h_{k v} \in S,\left(v \in I_{k}^{*}(x)\right)$ such that $m_{k} r_{k v}=h_{k v} t_{k v}$ for all $v \in I_{k}^{*}(x)$, and each $k \in I$.

Now we define a mapping $n_{1}: \Gamma \rightarrow \Gamma$ by putting

Clearly $n_{1} \in N$.

$$
\begin{gathered}
\left(\gamma_{v} . s\right) n_{1}=\gamma_{k}\left(h_{k v}\right) s \quad \text { for all } v \in I_{k}^{*}(x), k \in I, \\
0 . n_{1}=0,\left(\gamma_{\alpha} s\right) n_{1}=0 \text { for } \alpha \in X .
\end{gathered}
$$

Let $I^{\prime}=\left\{j \in I \mid \gamma_{i} x \in \gamma_{j} G\right.$ for some $\left.i \in I\right\}$. (Thus $I^{\prime}$ is the set of indices whose associated orbits appear in the image of $x$ in $\Delta$.)

Define $\theta: \Gamma \rightarrow \Gamma$ by

$$
\begin{array}{ll}
\gamma_{t} s \theta=\gamma_{t} m_{t} s & \text { for all } t \in I^{\prime}, \\
\gamma_{i} s \theta=\gamma_{i} s & \text { for all } i \in I \backslash I^{\prime}, \\
0 . \theta=0 . &
\end{array}
$$

Then $\theta$ is a regular element of $N$ and the element $\theta^{-1}: \Delta \rightarrow \Delta$ is given by

$$
\begin{array}{ll}
\gamma_{t} g \rightarrow \gamma_{t} m_{t}^{-1} g & \text { for all } t \in I^{\prime}, \\
\gamma_{i} g \rightarrow \gamma_{i} g & \text { for all } i \in I \backslash I^{\prime}
\end{array}
$$


Put $y=n_{1} \theta^{-1}$, then for $i \in I_{k}^{*}(x), \gamma_{i} y=\gamma_{i} n_{1} \theta^{-1}=\left(\gamma_{k} h_{k i}\right) \theta^{-1}=\gamma_{k} m_{k}^{-1} h_{k l}$ as $k \in I^{\prime}$. Now $m_{k} r_{k i}=h_{k i} t_{k i}$ and so in $G, r_{k i} t^{-1}=m_{k}^{-1} h_{k i}$. Thus $\gamma_{i} y=\gamma_{k} r_{k i} t_{k i}^{-1}=\gamma_{i} x$. If $j \in X$, then $\gamma_{j} y=\gamma_{j} n_{1} \theta^{-1}=0$ as $\gamma_{j} n_{1}=0$. Hence $y=x=n_{1} \theta^{-1}$. Thus $Q$ is a near-ring of right quotients of $N$.

Theorem 3.6. If $(\Gamma, S)$ is a 2-system, then $N=\operatorname{Map}_{S}(\Gamma)$ is a 2-prime near-ring.

Proof. Assume that $n, n^{\prime} \in N$ with $n N n^{\prime}=0$ but $n \neq 0$ and $n^{\prime} \neq 0$. Let $I=\{1,2, \ldots, p\}$. Since $n \neq 0$, then $\gamma_{i} n=\gamma_{j} s$ for some $i, j \in I$ and $s \in S$; also $\gamma_{t} n^{\prime}=\gamma_{r} s^{\prime}$ for some $t, r \in I$ and $s^{\prime} \in S$. Define $n_{1}: \Gamma \rightarrow \Gamma$ by

$$
\begin{array}{ll}
\gamma_{j} s_{1} n_{1}=\gamma_{t} s_{1} & \text { for any } s_{1} \in S, \\
\gamma_{1} s_{1} n_{1}=0 & \text { for any } s_{1} \in S \text { and } l \in I, l \neq j .
\end{array}
$$

Then $n_{1} \in N$ and $\gamma_{i} n n_{1} n^{\prime}=\gamma_{j} s n_{1} n^{\prime}=\gamma_{t} s n^{\prime}=\gamma_{\gamma} s^{\prime} s \neq 0$. But $n n_{1} n^{\prime} \in n N n^{\prime}=0$; thus we have a contradiction.

The theorems of this section show that if $(\Gamma, S)$ is a 2 -system then the nearring $\operatorname{Map}_{s}(\Gamma)$ will have a near-ring of right quotients which is in fact 2-primitive and artinian and is of the form $\operatorname{Map}_{G}(\Delta)$ for suitable groups $\Delta, G$. Notice that the near-ring of quotients, although an endomorphism near-ring is associated with the category of $G$-acts and $G$-morphisms rather than with the related category of $S$-acts and $S$-morphisms.

Some of the theorems of ring theory which describe the connection between a ring and its ring of right quotients (if it has one) can be generalised easily to the near-ring case. Using results of Betsch (1) concerning near-rings with d.c.c. on right ideals which have a zero $J_{2}$-radical, it is possible to prove that $N=\operatorname{Map}_{S}(\Gamma)$ has a.c.c. on right annihilators and possesses no infinite direct sums of right ideals (see (3)).

\section{REFERENCES}

(1) G. BETSCH, Struktursatze für Fastringe, Doctoral dissertation (EberhardKarls Universitat zu Tübingen, 1963).

(2) A. H. Clifford and G. B. Preston, The algebraic theory of semigroups, vols. I, II (Amer. Math. Soc. Publications, 1961, 1967). 1970).

(3) W. M. L. HoLcomBe, Primitive near-rings, Ph.D. Thesis (University of Leeds,

(4) U. KNauer and A. V. Mikhalev, Endomorphism monoids of acts over monoids, Semigroup Forum 6 (1973), 50-58.

(5) D. Ramakotaiah, Structure of 1-primitive near-rings, Math. Z. 110 (1969), 15-26.

(6) H. Wreland, Über Bereiche aus Gruppenabbildungen, Deutsche Math. 3 (1938), 9-10.

\section{QUEEN'S UNIVERSITY OF BeLFAST}

